\title{
Substrate/layer interface of amorphous-carbon hard coatings
}

\author{
O. Böhme $e^{\mathrm{a})}$ \\ Instituto de Ciencia de Materiales de Madrid, ICMM-CSIC, Cantoblanco, 28049 Madrid, Spain
}

\begin{abstract}
A. Cebollada
Instituto de Microelectrónica de Madrid, IMM (CNM-CSIC), C/Isaac Newton 8 (PTM), Tres Cantos, 28760 Madrid, Spain
\end{abstract}

S. Yang and D. G. Teer

Teer Coating Ltd., 290 Hartlebury Trading Estate, Hartlebury, Worcester DY1O 4JB, United Kingdom

J. M. Albella and E. Román

Instituto de Ciencia de Materiales de Madrid, ICMM-CSIC, Cantoblanco, 28049, Madrid, Spain

(Received 13 January 2000; accepted for publication 8 May 2000)

\begin{abstract}
A combined study of the crystalline structure, the chemical interaction, and diffusion processes of the substrate/layer interface of amorphous-carbon hard coatings is presented. The structure of the coatings and their gradient layer interface to a chromium buffer layer has been investigated on two substrates [ $\mathrm{Si}(100)$ and tool steel] using $\mathrm{x}$-ray diffraction (XRD). Chemical interaction and diffusion processes at the interfaces and within the layers were analyzed by Auger electron spectroscopy and x-ray photoemission spectroscopy depth profiles. The chromium buffer layer revealed similar textured structure on both substrates. The subsequent gradient layer was determined (within XRD limits) to be amorphous and composed of an amorphous-carbon and chromium-carbide composite. The chromium carbide maintains the same stoichiometry $\left(\mathrm{Cr}_{3} \mathrm{C}_{2}\right)$, regardless of the gradually changing chromium content. No large-scale interdiffusion was measured, either between or within the layers. (C) 2000 American Institute of Physics. [S0021-8979(00)01016-1]
\end{abstract}

\section{INTRODUCTION}

Due to their interesting applications as special coatings, diamond-like carbon films have attracted increasing interest in recent years. They are usually referred to as either amorphous-carbon $(a-\mathrm{C})$ films or hydrogenated-amorphouscarbon $(a-\mathrm{C}: \mathrm{H})$ films ${ }^{1}$ depending on the deposition technique. The exceptional properties of these films, such as extreme hardness, chemical inertness against acids and bases, optical transparency, etc., depend on the ratio of tetrahedral $\left(s p^{3}\right)$ bonded carbon to trigonal $\left(s p^{2}\right)$ bonded carbon and can vary over a wide range. Consequently, considerable efforts have been utilized to estimate the $s p^{3}$ to $s p^{2}$ ratio in relation to the film properties and various fabrication techniques and parameters.

However, for real coating applications, the film properties alone are often not the most important criteria for material selection. The properties of the substrate/layer combination of carbon-based coatings are some of the main factors to be considered in applications, as they determine the adherence of the coatings to components and tools and very often their thermal stability. ${ }^{2}$ In spite of the importance of the substrate/layer interface for technological applications, studies in this field, so far, are scarce. ${ }^{3-10}$

In this study, we focus on a substrate/layer design consisting of a highly adhesive metal buffer layer (chromium) which covers the substrate and converges homogeneously into the outer amorphous carbon $(a-\mathrm{C})$ layer. This gradient layer design is one of the concepts to approximate the requirements of "real coating" materials, such as good adher-

${ }^{\text {a)}}$ Electronic mail: boehme@icmm.csic.es ence at the substrate/layer interface and low interaction tendency at the surface, as well as high hardness and high toughness, which cannot be obtained simultaneously with the hard coating material alone., ${ }^{2,11}$ To date, there is no published work on whether an extended interface, consisting of a combined gradient metal $(\mathrm{Cr})$ and $a-\mathrm{C}$ layer, is maintained as deposited. This is essential information if one is to determine whether to use the more costly gradient layer interface in technological applications and will be studied in the present article. In addition to possible diffusion processes, the kind of chemical bonding in the gradient interface of $a-\mathrm{C}$ and $\mathrm{Cr}$ was studied. The adherence properties of $a$-C hard coatings are dependent on the formation of carbidic compounds at the substrate/layer interface. However, the presence of an interface carbide is not necessarily a guarantee of good adhesion as in the case of $a$-C/tungsten. ${ }^{3,6}$ To better understand the adherence properties between carbide-forming substrates and $a$ - $C$ coatings, the chemical information on whether a carbide has formed at the interface must be complemented by additional data about its structure. Interesting in this context is the structure that is formed by the combination of an amorphous material and a crystalline material. This is addressed for the carbidic phase of a reactive metal and amorphous carbon at the substrate/layer interface. The structure of the different layers has been analyzed by x-ray diffraction (XRD) and is combined with an Auger electron spectroscopy (AES) and a X-ray photoemission spectroscopy (XPS) analysis of the chemical interaction and diffusion processes therein. The combination of a $\mathrm{Cr}$ buffer layer, a $\mathrm{Cr}+\mathrm{C}$ gradient layer, and an $a-\mathrm{C}$ hard coating layer is not restricted to special substrates. It can be used to achieve good adherence 


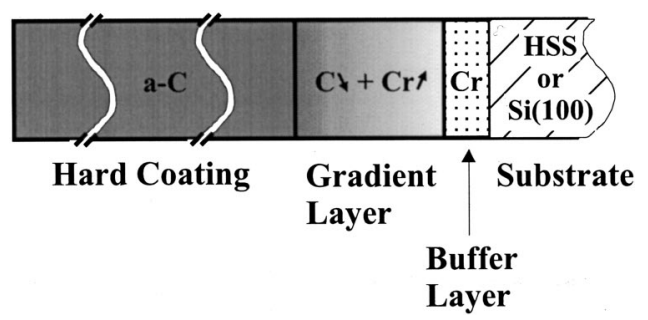

FIG. 1. Schematic view of the gradient layer design of amorphous carbon $(a-\mathrm{C})$ hard coatings.

properties $^{13,14}$ on a variety of substrates. In order to determine whether results obtained with coatings on "laboratory substrates" have similar characteristics in industrial applications, the influence of different substrate materials [Si(100) and HSS] on structure and chemistry of the coatings has been studied.

\section{EXPERIMENT}

The samples were prepared using a Teer UDP 450 unbalanced dc magnetron sputtering deposition system. ${ }^{12}$ The sequence of deposited elements during sample fabrication was expected to result in three different layers (Fig. 1): After an ion cleaning process, both M42 tool steel (HSS) and silicon $\mathrm{Si}(100)$ substrates were coated with a thin $(0.02 \mu \mathrm{m})$ chromium buffer layer which converges gradually over 0.18 $\mu \mathrm{m}$ into the amorphous-carbon hard coating. This outer carbon layer varies between $0.9 \mu \mathrm{m}$ (on $\mathrm{Si}$ substrates) and 1.5 $\mu \mathrm{m}$ (on the HSS substrates). These thicknesses have been estimated from the deposition time of the different stacks of layers. The deposition parameters are given in more detail elsewhere. ${ }^{13}$

The XRD measurements were recorded on a Philips MRD X'Pert diffractometer using $\mathrm{Cu} K \alpha$ radiation $(\lambda$ $=1.5418 \AA$ ) in the Bragg Brentano configuration. The XPS and AES measurements were performed in an ultra-highvacuum chamber with a base pressure of $1 \times 10^{-10} \mathrm{mbar}$, using a Perkin-Elmer ESCA/Auger spectrometer with double-pass cylindrical mirror analyzer. The AES data were acquired in the normal mode. The kinetic energy of the incident electron beam was $3 \mathrm{keV}$ with a total current of about $30 \mathrm{nA}$. The XPS measurements were performed using $\operatorname{Mg} K \alpha$ radiation and a pass energy of $50 \mathrm{eV}$.

Depth profiling was performed with $3 \mathrm{keV}$ argon ions over an area of $6 \times 10 \mathrm{~mm}^{2}$ and a total sample current of 4.4 $\mu \mathrm{A}$. The depth resolution of the profiling is given by the lateral inhomogenity of the ion-etching process and was measured (at a sharp interface at a depth of $1 \mu \mathrm{m}$ ) to be around $5 \%$ of the respective depth.

\section{RESULTS AND DISCUSSION}

\section{A. Chemical characterization of amorphous carbon on silicon substrates}

Studies on industrial $a$-C hard coatings with optimized tribological performance ${ }^{13,14}$ are presented in this work. The elemental composition of a sample deposited on $\mathrm{Si}(100)$ can be seen in the AES depth profile in Fig. 2. In order to obtain

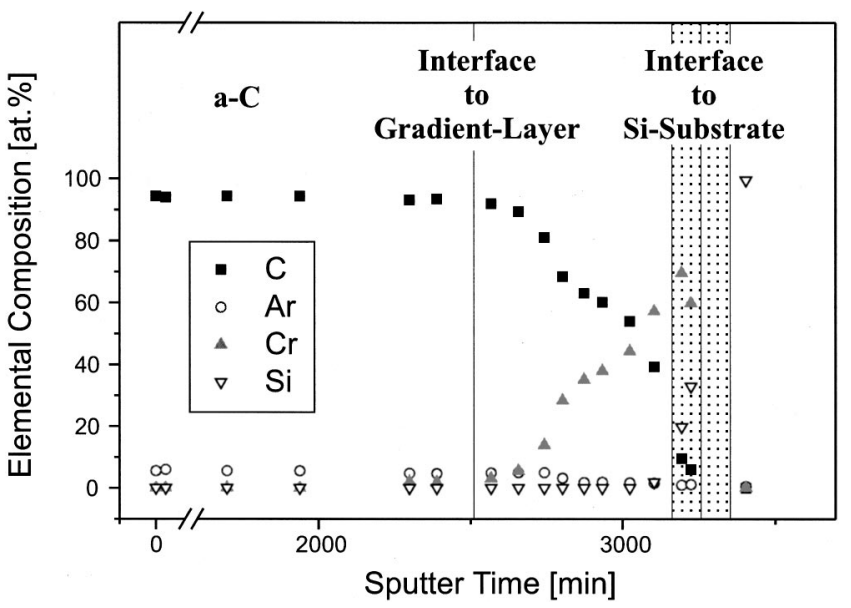

FIG. 2. AES depth profile of an $a$-C/Si(100) sample. The dotted region surrounding the $\mathrm{Si}$ Interface represents the resolution of the profiling process at a depth of about $1 \mu \mathrm{m}$.

evidence about possible diffusion processes, the real sample composition shown in the depth profile (Fig. 2) should be compared to the composition which is expected from the sequence of deposited elements during preparation (Fig. 1). The surface composition of the untreated sample was $88.6 \%$ carbon, $6.2 \%$ argon, and $5.2 \%$ oxygen. The presence of oxygen at the surface is due to surface contamination and is removed after a short Ar-ion bombardment (excluded from the time axis of the depth profile). Thus, after the ion cleaning, the composition of the sample is $94.0 \%$ carbon and $6.0 \%$ argon. This composition remains constant within $\pm 0.5 \%$ throughout the whole outer $a$-C layer (up to $2300 \mathrm{~min}$ of the sputter time). It should be mentioned that the profiling parameters used normally lead to an implantation of argon but never exceed $6 \%$, as has been shown for various $a$-C coatings with different initial $\mathrm{Ar}$ content. The $a$-C layers presented in this study had an initial Ar content of about 6\% and did not show any further increase of Ar by the profiling process.

Just before reaching the interface of the $a$-C layer to the gradient layer, there is already a small zone (between 2300 and $2550 \mathrm{~min}$ of the sputter time) with a constant chromium content of 2\% (as well as 93\% carbon and 5\% argon). This small zone is followed (up to $3200 \mathrm{~min}$ of the sputter time) by the $\mathrm{Cr}+\mathrm{C}$ gradient layer wherein the carbon content decreases almost linearly below $10 \%$ while the chromium content increases almost linearly up to $70 \%$. Thereafter, the chromium content decreases to $0 \%$ at the same rate as the silicon content increases to $100 \%$ (at about $3350 \mathrm{~min}$ of the sputter time). Concerning the coating procedure of the samples, beneath the $\mathrm{Cr}+\mathrm{C}$ gradient layer there should be a pure $\mathrm{Cr}$ buffer layer of $0.02 \mu \mathrm{m}$ thickness. However, in a profiling depth of about $1 \mu \mathrm{m}$ the thickness of this buffer layer is already beyond the resolution of the profiling process.

As a first approximation, neglecting the influence of different materials and compositions on the sputter rate, the average sputter rate can be estimated as $0.33 \mathrm{~nm} / \mathrm{min}$. Using this sputter rate, the respective times needed to remove the different layers correlate with the thickness specifications ob- 


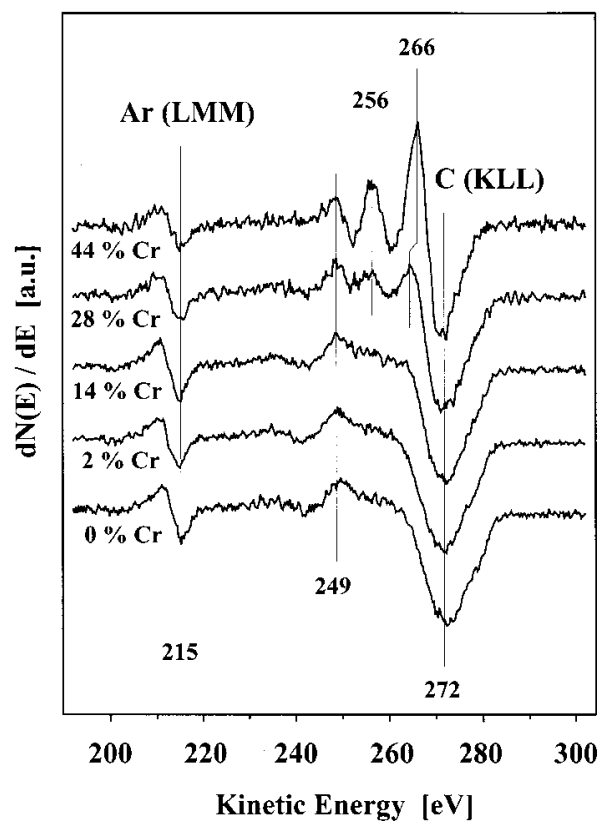

FIG. 3. Evolution of the Auger $\mathrm{C}(K L L)$ line shape with increasing $\mathrm{Cr}$ content in the gradient layer of an $a-\mathrm{C} / \mathrm{Si}(100)$ sample.

tained during sample preparation. Apart from the small zone of the $a$-C layer preceding the gradient layer, the elemental composition of the respective depth coincides with the given specifications of the preparation process. Thus, large-scale diffusion does not occur at the interfaces and within the different layers. The gradient of $\mathrm{Cr}$ and $\mathrm{C}$ within the intermediate gradient layer is maintained as deposited, which is of special interest with regard to the adherence properties of the film. On a smaller scale, diffusion processes cannot be excluded. Nevertheless, the constant and low $\mathrm{Cr}$ content of about $2 \%$ in front of the gradient layer does not resemble a diffusion zone. Its origin is thought to be rather found in the deposition process.

An interesting aspect of the substrate/layer design presented here is the chemical interaction within the $\mathrm{Cr}+\mathrm{C}$ gradient layer. Qualitative insight can be obtained by line-shape analysis of the AES carbon $K L L$ transition and its evolution with increasing chromium content upon depth profiling. Figure 3 shows Auger spectra normalized to the negative excursion of the $\mathrm{C}(K L L)$ main transition. The $\operatorname{Ar}(L M M)$ transition was used for energy calibration and was set to 215 $\mathrm{eV} .{ }^{15}$ The lower spectrum of Fig. 3 was taken at the beginning of the depth profiling procedure $(0 \mathrm{~min})$ and corresponds to the $a-\mathrm{C}$ layer without any $\mathrm{Cr}$ contribution. The $\mathrm{C}$ $(K V V)$ transition of this spectrum reveals a main transition at $272 \mathrm{eV}$ and a fine structure at lower energies, consisting of a pronounced transition at $249 \mathrm{eV}$ and a less pronounced shoulder at approximately $256 \mathrm{eV}$. The fine structure between 240 and $260 \mathrm{eV}$ in the Auger spectra of $a-\mathrm{C}$ films is usually compared to those of diamond and graphite. In the case of diamond they are exclusively assigned to $\sigma^{*} \sigma$ folds, while in the case of graphite one (at lower energies) is assigned to the $\sigma^{*} \sigma$ fold and another (at higher energies) is assigned to the $\sigma^{*} \pi$ fold. ${ }^{16}$ The main Auger transition can be either compared to the $\pi^{*} \pi$ fold of graphite $(272 \mathrm{eV})$ or to

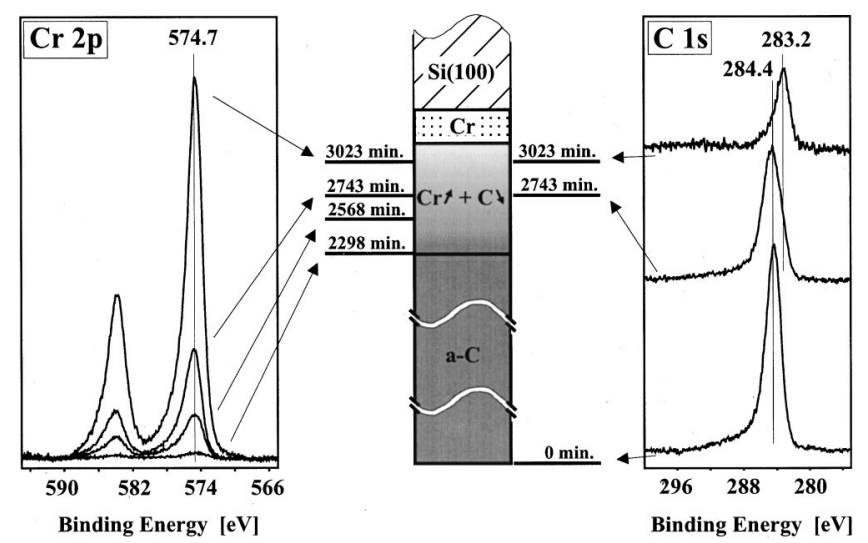

FIG. 4. XPS ( $\operatorname{Mg} K \alpha) \mathrm{Cr} 2 p$ and $\mathrm{C} 1 s$ core-level spectra of an $a$-C/Si(100) sample. The spectra have been taken at increasing depths (mainly in the gradient layer) with increasing $\mathrm{Cr}$ content.

the $\sigma_{p}^{*} \sigma_{p}$ fold of diamond $(267 \mathrm{eV})$. The sample has already been subject to a sputter cleaning procedure, which although short, led to an increase of the $s p^{2}$ hybridized carbon and a more graphite-like aspect of the line shape.

With increasing chromium content in the $\mathrm{Cr}+\mathrm{C}$ gradient layer, the $\mathrm{C}(K L L)$ spectra change drastically until, in the upper spectrum in Fig. 3, the line shape corresponds completely to a typical transition-metal carbide spectrum. The three local maxima $(249,256$, and $266 \mathrm{eV})$ can be regarded as a fingerprint for transition-metal carbon bonding (not restricted to chromium carbide) and the relation of their respective intensities allows a quantification of the percentage of carbon that has reacted to a transition-metal carbide. ${ }^{17} \mathrm{In}$ the upper spectrum, which represents a part of the gradient layer with $44 \%$ of $\mathrm{Cr}$, carbon is mainly present as chromium carbide. The outer parts of the gradient layer (i.e., with lower Cr content) consist of a composite of carbon and chromium carbide which gives rise to linearly combined $a-\mathrm{C}$ and chromium carbide spectra between the lower and upper spectrum of Fig. 3.

Additional information about the chemical bonding within the film, as well as the stoichiometry of the chromium carbide in the gradient layer, has been obtained using XPS. In Fig. 4 the $\mathrm{Cr} 2 p$ and the $\mathrm{C} 1 s$ core-level spectra can be seen at increasing depth (increasing $\mathrm{Cr}$ content) of the film. The $\mathrm{Cr} 2 p$ spectra span a small zone of the $a$-C layer (with $2 \%$ chromium) and the whole gradient layer region. No chemical shift of the $\mathrm{Cr}$ peak was observed, indicating that the chemical bonding state of the chromium does not change with increasing $\mathrm{Cr}$ content in the film. The $\mathrm{C} 1 s$ spectra shown in Fig. 4 have been chosen for the outer $(2743 \mathrm{~min}$ ) and the inner (3023 min) part of the gradient layer. For comparative purposes, a spectrum of the pure $a-\mathrm{C}$ layer is shown below. Compared to this lower spectrum, the main component of the $\mathrm{C} 1 \mathrm{~s}$ peak in the upper spectrum is shifted $1.2 \mathrm{eV}$ to lower binding energies. In this part of the gradient layer almost all carbon has reacted to form chromium carbide. The elemental ratio of $\mathrm{Cr}: \mathrm{C}=1.66: 1$ was calculated from peak area measurements using tabulated atomic sensitivity factors ${ }^{18}$ and indicates the formation of $\mathrm{Cr}_{3} \mathrm{C}_{2}$. Only the component at $283.2 \mathrm{eV}$, which forms $90 \%$ of the carbon 

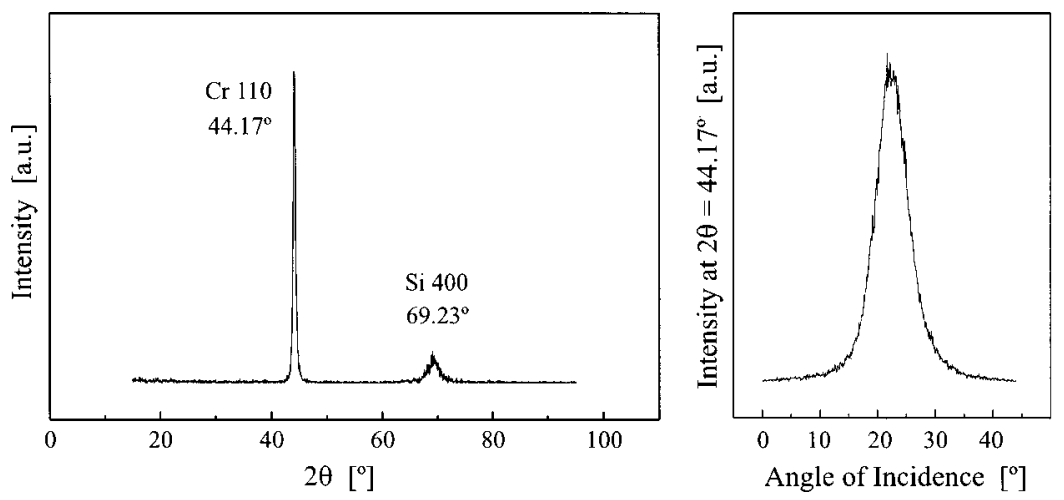

FIG. 5. XRD $(\mathrm{Cu} K \alpha)$ spectra of an $a-\mathrm{C} / \mathrm{Si}(100)$ sample. Left: $\theta / 2 \theta$ scan, revealing the family of planes parallel to the sample surface. Right: rocking curve of the $\mathrm{Cr} 110$ planes. peak in the upper spectrum, has been used to calculate the peak area. The intermediate spectrum shows both the main component of the lower spectrum (at $284.4 \mathrm{eV}$ ) and the main component of the upper spectrum (at $283.2 \mathrm{eV}$ ). This coincides with the formation of a carbon and chromium-carbide composite in the gradient layer, which has already been demonstrated in the AES results above. In this study, we focused on the chromium-carbide component $(283.2 \mathrm{eV})$ of the carbon peak and the amorphous carbon was represented only by its main component at $284.4 \mathrm{eV}$. This latter component originates from carbon atoms in the $s p^{2}$ configuration, ${ }^{19,20}$ which in our case, and especially after the ion bombardment, forms the major part of the carbon.

\section{B. Structural characterization of amorphous carbon on silicon substrates}

The crystalline structure of the samples was obtained by XRD measurements. The XRD spectra of the coating prepared on the silicon substrate are presented in Fig. 5. A $\theta / 2 \theta$ scan (Fig. 5, left) reveals the family of planes parallel to the sample surface without any depth dependance. The measurement was done with the substrate slightly misaligned. This only affects the intensity of the monocrystalline Si substrate peaks; film signals are not affected because of their larger width (see the rocking curve). In addition to the $\mathrm{Si} 400$ peak at $2 \theta=69.23^{\circ}$, a pronounced and narrow peak at $2 \theta=44.17^{\circ}$ is observed. This peak has been identified as the $\mathrm{Cr} 110$ peak from the thin chromium buffer layer at the interface to the silicon substrate. There are a variety of other possible crystal planes in the vicinity of $2 \theta=44.2^{\circ}$, e.g., graphite 101 , diamond 111 , as well as some chromium carbides. Nevertheless, its identification as $\mathrm{Cr} 110$ is unambiguous, since XRD measurements realized with the mere chromium layer on the silicon substrate (not shown here) reveal exactly the same spectra $\left(\theta / 2 \theta\right.$ and rocking curve). The displacement of $\Delta_{2 \theta}$ $=0.22^{\circ}$ of the $\mathrm{Cr} 110$ peak relative to standard polycrystalline chromium is due to stress in this layer and corresponds to an enlargement from 2.036 to $2.050 \AA$ of the $\mathrm{Cr}$ bonding length in the 110 direction, i.e., $0.7 \%$ expansion. A possible cause of this expansion may be the presence of interstitial argon (about $2 \%$ ) originating from the film preparation process by unbalanced dc magnetron sputtering. The chromium buffer layer shows a preferential orientation of the 110 planes parallel to the sample surface. The mosaic spread (angular variation respective to the sample surface) can be taken from the rocking curve of the $\mathrm{Cr} 110$ peak (Fig. 5, right) to be $\pm 5^{\circ}$.

The intermediate $\mathrm{Cr}+\mathrm{C}$ gradient layer does not show strong crystalline features. The XRD spectra of a mere $\mathrm{Cr}+\mathrm{C}$ gradient layer (representative for the intermediate layer in the $a$-C samples) deposited on a silicon substrate is presented in Fig. 6. The weak signal at $2 \theta=43.85^{\circ}$ in the $\theta / 2 \theta$-spectrum (Fig. 6, left) was assigned to the already known Cr 110 signal, and is due to the small amount of nearly pure chromium at the beginning of the $\mathrm{Cr}+\mathrm{C}$ ramp. Due to the reduced thickness of the pure chromium buffer layer in this sample, there is more stress, which leads to a higher displacement of $\Delta_{2 \theta}=0.54^{\circ}$ of the $\operatorname{Cr} 110$ peak, i.e., $1.4 \%$ expansion of the $\mathrm{Cr}$ bonding in the 110 direction. We have not checked a possible additional contribution to the increase of stress due to the argon content at the beginning of the ramp.

The outer $a$-C hard coating layer itself shows no crystalline features. This has been proven with grazing-incidence XRD measurements (not shown here) at incidence angles of
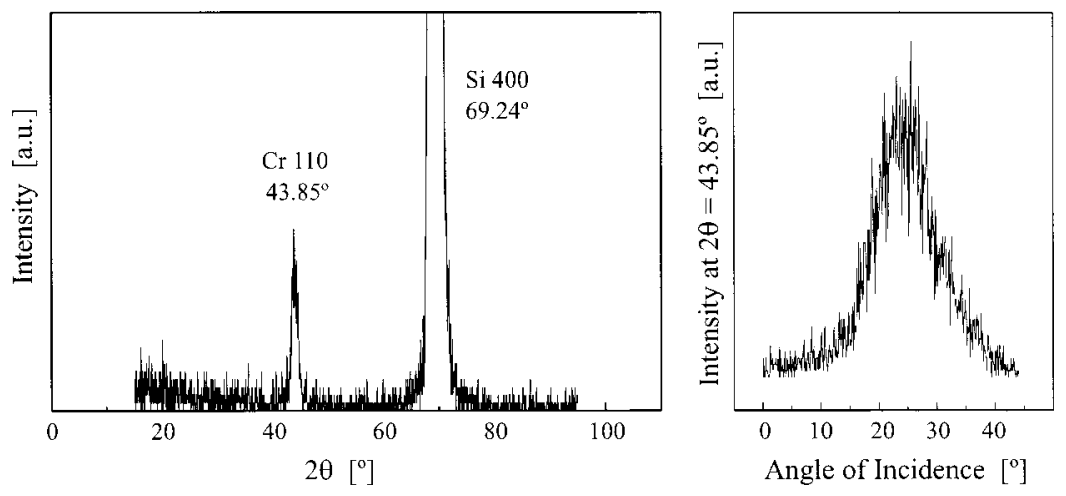

FIG. 6. XRD $(\mathrm{Cu} K \alpha)$ spectra of a sample which only consists of the $\mathrm{Cr}+\mathrm{C}$ gradient layer on $\mathrm{Si}(100)$. Left: $\theta / 2 \theta$ scan and right: rocking curve of the weak $\mathrm{Cr} 110$ peak. 


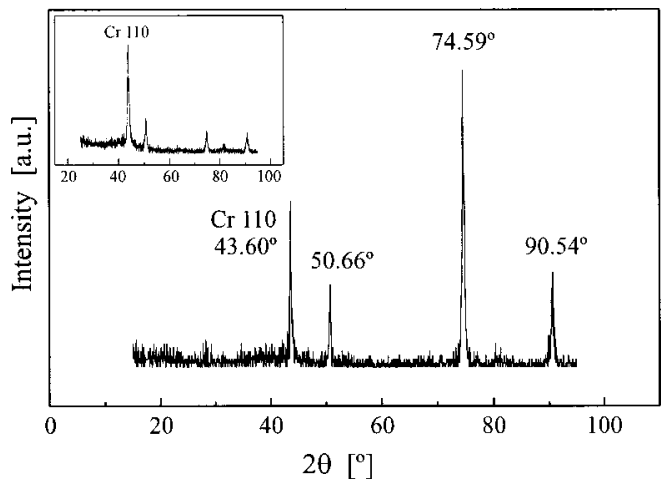

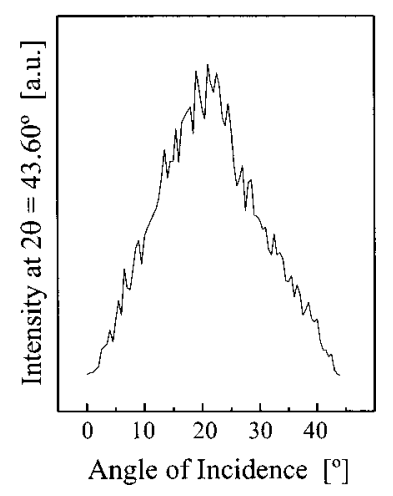

FIG. 7. XRD $(\mathrm{Cu} K \alpha)$ spectra of an $a$-C/HSS sample. Left: $\theta / 2 \theta$ scan, presenting the family of planes without any depth dependence. The inset presents the more surface sensitive grazing-incidence spectrum, taken at an incidence angle of $5^{\circ}$. Right: rocking curve of the $\mathrm{Cr}$ 110 planes. $\theta=1^{\circ}$ and long integration times. At an incidence angle of $1^{\circ}$ the whole depth of the outer $a$-C coating is already analyzed and absorbs about $10 \%$ of the incident radiation.

It is worth mentioning that no crystalline features due to chromium carbide were observed in the XRD spectra, although the AES and XPS results clearly indicate that in a typical $a$ - $\mathrm{C}$ sample, all the chromium in the $\mathrm{Cr}+\mathrm{C}$ gradient layer reacts to chromium carbide. This implies that either the crystallite size of the chromium carbide in the gradient layer is smaller than the detection limit of XRD, or that the chromium carbide is amorphous. Our findings deviate from a recently published study which states a crystalline $\mathrm{Cr}_{7} \mathrm{C}_{3}$ phase in Cr-containing $a-\mathrm{C}: \mathrm{H}$ films. ${ }^{21}$ The reason for this difference can be found in the deposition technique (plasmaactivated chemical-vapor deposition) that was used. It is interesting to compare the XPS measurements of the cited reference with those presented in Fig. 4: The higher coordinated carbon in the $\mathrm{Cr}_{7} \mathrm{C}_{3}$ phase consistently shows an increase of about $0.5 \mathrm{eV}$ in the chemical shift towards lower binding energies compared to the carbon in the $\mathrm{Cr}_{3} \mathrm{C}_{2}$ phase present in our samples.

\section{Structural characterization of amorphous carbon on HSS substrates}

In the substrate/layer design presented in this study, the choice of substrate ( $\mathrm{Si}, \mathrm{HSS}$ ) can only influence the outer hard coating via the metal buffer layer and the gradient layer. However, the metal buffer layer already shows the same crystalline structure on the HSS substrates as on the Si substrates. The chromium buffer layer on HSS shows the same preferential orientation in the 110 direction, as indicated by the $\mathrm{Cr} 110$ peak at $43.60^{\circ}$ in the $\theta / 2 \theta$ spectrum of Fig. 7. The displacement of the $\mathrm{Cr} 110$ peak is $\Delta_{2 \theta}=0.79^{\circ}$, which corresponds to a $2.0 \%$ expansion of the $\mathrm{Cr}$ lattice spacing. Also in this case, the difference in the bonding expansion (cf. films on Si samples) is considered to be a consequence of a slightly varying amount of interstitial argon in the buffer layer. Nevertheless, the industrial samples used in the present study did not allow an exact measurement of the argon content in the buffer layer as it cannot be resolved by depth profiling. Thus, referring to the stress in the buffer layer, we cannot separate the effect of interstitial argon from the influence of the substrates.

The other peaks in the $\theta / 2 \theta$ spectrum of Fig. 7 originate from the HSS substrate underneath the Cr buffer, which can be clearly seen when compared to the grazing-incidence XRD measurement presented in the inset. This grazingincidence spectrum was recorded using an incidence angle of $5^{\circ}$ and shows the relative increase of the $\mathrm{Cr} 110$ peak when the measurement becomes more surface sensitive. There is an additional small peak at $2 \theta=81.33^{\circ}$ in the grazingincidence spectrum. This peak can be attributed to the $\mathrm{Cr} 211$ planes of the Cr buffer layer. In a polycrystalline sample this Cr 211 peak would contribute with $30 \%$ of the $\mathrm{Cr} 110$ peak intensity. In the textured $\mathrm{Cr}$ buffer of the present sample, it only appears very damped.

The mosaic spread of the preferentially oriented 110 planes of the $\mathrm{Cr}$ buffer can be taken from the rocking curve in Fig. 7 to be $\pm 10^{\circ}$. This is twice the value obtained for the $\mathrm{Cr}$ buffer layer on silicon substrates. The reason for the higher angular variation may be found in the lower planarity of the HSS substrate surface; reflecting the variation respective to a virtual average planar surface.

\section{CONCLUSIONS}

The substrate/layer interface of $a-\mathrm{C}$ hard coatings, optimized respective to their tribological properties, was analyzed using XRD in combination with AES and XPS depth profiles. Within the detection limits of XRD, both the outer $a-\mathrm{C}$ hard coating layer, as well as the intermediate carbon chromium gradient layer, showed an amorphous structure. They have grown over a thin chromium buffer layer which shows the same texture in the 110 direction, regardless of substrate type [Si(100) and HSS] used.

The chromium buffer layer continually converges within the gradient layer into the amorphous-carbon hard coating layer. All the chromium of the gradient layer has formed as chromium carbide. Although the chromium content gradually changes within this layer, the chromium carbide maintains the same stoichiometry $\left(\mathrm{Cr}_{3} \mathrm{C}_{2}\right)$. The gradient layer is thus formed by a composite of $a-\mathrm{C}$ and $\mathrm{Cr}_{3} \mathrm{C}_{2}$ with successively varying proportion. The chromium and the carbon gradient within this layer is maintained as deposited. There are no large-scale diffusion processes at the interfaces and within the different layers.

\section{ACKNOWLEDGMENTS}

Financial support by the European Community within the BRITE-EURAM III Program under Contract No. 
BRPR-CT97-0487 is gratefully acknowledged. One of the authors (O.B.) gratefully acknowledges financial support by the European Community under Grant No. BRMA-CT985136.

${ }^{1}$ J. Robertson and E. P. O'Reilly, Phys. Rev. B 35, 2946 (1987).

${ }^{2}$ H. Holleck, J. Vac. Sci. Technol. A 4, 2661 (1986).

${ }^{3}$ R. Zehringer, R. Hauert, and M. Tobler, Thin Solid Films 208, 38 (1992).

${ }^{4}$ R. Hauert, J. Patscheider, M. Tobler, and R. Zehringer, Surf. Sci. 292, 121 (1993).

${ }^{5}$ T. Bruce, I. Bello, L. J. Huang, W. M. Lau, M. High, V. Stmad, and P. Panchhi, J. Appl. Phys. 76, 552 (1994).

${ }^{6}$ K. H. Ernst, J. Patscheider, R. Hauert, and M. Tobler, Surf. Interface Anal. 21, 32 (1994).

${ }^{7}$ C. A. Davis, K. M. Knowles, and G. A. J. Amaratunga, Surf. Coat. Technol. 76, 316 (1995).

${ }^{8}$ J. Schafer, J. Ristein, S. Miyazaki, and L. Ley, J. Vac. Sci. Technol. A 15, 408 (1997).

${ }^{9}$ S. Logothetidis, E. Evangelou, and N. Konofaos, J. Appl. Phys. 82, 5017 (1997).

${ }^{10}$ C. Tixier, M. Cahoreau, and J. Desmaison, Key Eng. Mater. 132, 1540 (1997).
${ }^{11}$ H. Holleck, Vacuum 41, 2220 (1990)

${ }^{12}$ D. G. Teer, Patent No. 225843A (United Kingdom).

${ }^{13}$ S. Yang, D. Camino, A. H. S. Jones, and D. G. Teer, Surf. Coat. Technol. 124, 110 (2000).

${ }^{14} \mathrm{~S}$. Yang and D. G. Teer, presented at The Asia/Europe Plasma Surface Engineering Conference (AEPSE 99), Beijing. P. R. China, September 1999.

${ }^{15}$ L. E. Davis, N. C. MacDonald, P. W. Palmberg, G. E. Riach, and R. E. Weber, Handbook of Auger Electron Spectroscopy, 2nd ed. (Physical Electronics, Eden Prairie, MN, 1976).

${ }^{16}$ H. J. Steffen, C. D. Roux, D. Marton, and I. W. Rabalais, Phys. Rev. B 44, 3981 (1991).

${ }^{17}$ S. Craig, G. L. Harding, and R. Payling, Surf. Sci. 124, 591 (1983).

${ }^{18}$ C. D. Wagner, W. M. Riggs, L. E. Davis, J. F. Moulder, and G. E. Muilenberg, Handbook of X-Ray Photoelectron Spectroscopy (Perkin-Elmer, Eden Prairie, MN, 1979).

${ }^{19}$ J. Diaz, G. Paolicelli, S. Ferrer, and F. Comin, Phys. Rev. B 54, 8064 (1995).

${ }^{20}$ T. Y. Leung, W. F. Man, P. K. Lim, W. C. Chan, G. Gaspari, and S. Zukotnynski, J. Non-Cryst. Solids 254, 156 (1999).

${ }^{21}$ P. Gantenbein, S. Brunold, U. Frei, J. Geng, A. Schüler, and P. Oelhafen, Carbon 37, 843 (1999). 